\title{
Anti-inflammatory Effects of Novel Polysaccharide Sacran Extracted from Cyanobacterium Aphanothece sacrum in Various Inflammatory Animal Models
}

\author{
Keiichi Motoyama, ${ }^{a}$ Yuki Tanida,${ }^{a}$ Kyona Hata,${ }^{a}$ Tomoya Hayashi,${ }^{a}$ Irhan Ibrahim Abu Hashim,,${ }^{a, b}$ \\ Taishi Higashi, ${ }^{a}$ Yoichi Ishitsuka, ${ }^{a}$ Yuki Kondo, ${ }^{a}$ Tetsumi Irie, ${ }^{a, c}$ Shinichiro Kaneko, ${ }^{d}$ and \\ Hidetoshi Arima ${ }^{*, a, c}$ \\ ${ }^{a}$ Graduate School of Pharmaceutical Sciences, Kumamoto University; 5-1 Oe-honmachi, Chuo-ku, Kumamoto \\ 862-0973, Japan: ${ }^{b}$ Faculty of Pharmacy, Mansoura University; Mansoura 35516, Egypt: ${ }^{c}$ Program for Leading \\ Graduate Schools "HIGO (Health life science: Interdisciplinary and Glocal Oriented) Program," Kumamoto \\ University; 5-1 Oe-honmachi, Chuo-ku, Kumamoto 862-0973, Japan: and ${ }^{d}$ Green Science Material Inc.; 4-12-25 \\ Nagamine-higashi, Higashi-ku, Kumamoto 861-8038, Japan. \\ Received February 29, 2016; accepted April 26, 2016; advance publication released online May 10, 2016
}

\begin{abstract}
The goal of this study was to investigate the topical anti-inflammatory effects of the megamolecular polysaccharide sacran extracted from cyanobacterium Aphanothece sacrum using various inflammatory animal models. Sacran showed potent anti-inflammatory effects with optimum effective concentrations at 0.01 and $0.05 \%(w / v)$. Sacran markedly inhibited paw swelling and neutrophil infiltration in carrageenan-induced rat paw edema. Additionally, 6,7-dimethoxy-1-methyl-2(1H)-quinoxalinone-3-propionyl-carboxylic acid (DMEQ)-labeled sacran had the ability to penetrate carrageenan-induced rat paw skin rather than normal skin. Also, sacran significantly suppressed kaolin-induced and dextran-induced rat paw edema throughout the duration of the study. Furthermore, sacran significantly suppressed 12- $O$-tetradecanoylphorbol-13-acetate (TPA)-induced mouse ear edema and mRNA expression levels of cyclooxygenase (COX)-2 as well as pro-inflammatory cytokines such as tumor necrosis factor (TNF)- $\alpha$, interleukin (IL)-1 $\beta$, and IL-6. Safety of sacran solution was verified by negligible cytotoxicity in HaCaT cells. These results suggest that sacran may be useful as a therapeutic agent against inflammatory skin diseases with no life-threatening adverse effects.
\end{abstract}

Key words sacran; polysaccharide; anti-inflammatory effect; phlogistic agent

Inflammatory responses are complex events of the body as it delivers the appropriate defense against harmful stimuli. Inflammation is partially regulated by carbohydrates, especially sulfated glycosaminoglycans, expressed on the surface of endothelial cells and leukocyte cells. Exogenous sulfated glycans, such as heparin, heparan sulfate, dermatan sulfate, chondroitin sulfate, fucosylated chondroitin sulfate and fucoidans can induce anti-inflammatory effects. The anti-inflammatory effects of these sulfated glycans are the competitive inhibition of the molecular interactions between sulfated glycans and P-selectin or L-selectin, while other mechanisms of action, such as down-regulation of chemokine and transcription factor activities can also occur.

Skin operates not only as a protective physical barrier, but also as a dynamic organ that has other recognized functions, such as endogenous homeostasis, metabolism, and sensory input. Moreover, skin actively contributes in immunological regulatory processes and inflammatory responses. ${ }^{1)}$ There is a wide range of dermatological conditions that include inflammatory skin disorders ranging in severity from mild skin rash to severe dermatitis. Nowadays, corticosteroids and nonsteroidal anti-inflammatory drugs (NSAIDs) are commonly administered to reduce the inflammation. However, they can frequently cause a number of serious adverse effects., ${ }^{2,3}$ Hence, great efforts have been devoted towards the discovery of new and safe anti-inflammatory natural products of plant origin as alternatives. ${ }^{4,5)}$ Importantly, numerous beneficial effects of plant polysaccharides have been demonstrated on human health to exhibit a spectrum of biological activities such as anti-inflammatory effects. ${ }^{6,7)}$ Meanwhile, plant polysaccharides may have the potential to induce the allergic response. Therefore, plant polysaccharides with high safety are necessary.

Recently, a novel sulfated polysaccharide sacran attracts a considerable amount of attention. It is extracted from the Japanese indigenous cyanobacterium Aphanothece sacrum, which possesses a jelly-like extracellular matrix (ECM) with high water content $(97.5-98.3 \%){ }^{8-12)}$ Sacran is a heteropolysaccharide composed of various sugar residues such as glucose, galactose, mannose, xylose, rhamnose, fucose, galacturonic acid and glucuronic acid, and contains traces of alanine, galactosamine and muramic acid; $11 \%$ of monosaccharides contain a sulfate group and $22 \%$ of them contain a carboxyl group. In addition, sacran was reported to be a supergiant molecule with extremely high molecular weight ranging over $10^{7} \mathrm{~g} / \mathrm{mol}$ and micrometer-scaled (more than $8 \mu \mathrm{m}$ ). Previously, Ngatu et al. revealed that sacran has anti-inflammatory effects on 2,4,6-trinitrochlorobenzene-induced allergic dermatitis model mice. ${ }^{13)}$ So far, however, other inflammatory models have not yet been examined. Therefore, in the present study, we focused on the investigation of the anti-inflammatory effects of sacran on various experimental animal models of inflammation.

\section{MATERIALS AND METHODS}

Materials Sacran was extracted from Aphanothece sacrum by Green Science Material (Kumamoto, Japan), as 
reported previously.,11) $\lambda$-Carrageenan and 6,7-dimethoxy1-methyl-2(1H)-quinoxalinone-3-propionyl-carboxylic acid (DMEQ)-hydrazide were purchased from Wako Pure Chemical Industries, Ltd. (Osaka, Japan). Kaolin, dextran, 4-biphenyl acetic acid (BPAA) and prednisolone (PD) were obtained from Nacalai Tesque (Kyoto, Japan). 12-O-Tetradecanoylphorbol13-acetate (TPA) was purchased from Sigma-Aldrich (Tokyo, Japan). HaCaT cells, a human epidermal keratinocyte line, were purchased from Deutsches Krebsforschungszentrum (DKFZ; German Cancer Research Center, Cell Lines Service, Eppelheim, Germany). All other chemicals and solvents were of analytical reagent grade.

Animals Male Wistar rats (8 weeks old) and female BALB/c mice (5 weeks old) were purchased from SLC (Shizuoka, Japan). They were maintained under controlled conditions $\left(22^{\circ} \mathrm{C}, 55 \%\right.$ humidity and $12 \mathrm{~h}$ day/night rhythm) and fed a standard laboratory chow. Animal studies were approved by the Ethics Committee for Animal Care and Use of Kumamoto University.

\section{In Vivo Anti-inflammatory Activity}

Carrageenan-Induced Rat Paw Edema

The rats received a subplantar injection of $\lambda$-carrageenan $(100 \mu \mathrm{L}, 1 \%$ (w/v) in normal saline) in the right hind paw. One hour later, sacran solutions with various concentrations $(0.01,0.05,0.1 \%(\mathrm{w} / \mathrm{v}))$ were topically administered every $1 \mathrm{~h}$ after carrageenan injection. The rats in the negative control group were treated with normal saline solution. ${ }^{14)}$ The animals of positive control group were treated with BPAA as a reference anti-inflammatory drug. The paw volume of each animal was determined before carrageenan injection, and then hourly intervals until $6 \mathrm{~h}$ after induction of inflammation, with a plethysmometer. The increase in the paw volume was calculated by subtracting the initial paw volume to the paw volume measured at each time interval.

\section{Kaolin-Induced Rat Paw Edema}

The animals were treated in a similar manner to that of carrageenan-induced rat paw edema model; kaolin $(100 \mu \mathrm{L}, 20 \%$ $(w / v)$ in normal saline) was used instead of $\lambda$-carrageenan.

Dextran-Induced Rat Paw Edema

The treatment of animals and measurements of the paw volume were performed as described above. Dextran $(100 \mu \mathrm{L}$, $1 \%(\mathrm{w} / \mathrm{v})$ in normal saline) was used as an inducer of rat paw edema.

TPA-Induced Mouse Ear Edema

Ear edema was induced in mice by topical application of $3 \mu \mathrm{L}$ of TPA $(1 \mathrm{mg} / \mathrm{mL})$ and then $40 \mu \mathrm{L}$ of sacran solutions $(0.01,0.05,0.1,1.0 \%(\mathrm{w} / \mathrm{v}))$ were administered to the opposite site of TPA-treated ear. Edema was expressed as the difference between the basal ear thickness and the ear thickness after $6 \mathrm{~h}$ of TPA application using dial thickness gauge (Teclock Corporation, Nagano, Japan).

Hematoxylin-Eosin (H\&E) Staining of CarrageenanInduced Acute Edema in Rat Paws For histological analysis, a paw tissue was resected $3 \mathrm{~h}$ after subplantar injection of $\lambda$-carrageenan solution $(100 \mu \mathrm{L}, 1 \%$ (w/v) in normal saline). The samples were fixed in $4 \%(\mathrm{w} / \mathrm{v})$ paraformaldehyde, embedded in paraffin, sectioned at thickness of $8 \mu \mathrm{m}$ and stained with H\&E for light microscopy analysis.

Skin Permeability of DMEQ-Labeled Sacran in Carrageenan-Induced Rat Paw Edema Model DMEQ-labeled sacran solution $(0.05 \%(\mathrm{w} / \mathrm{v}))$ was topically administered every $1 \mathrm{~h}$ after subplantar injection of $\lambda$-carrageenan to a rat paw. Three hours later, resected segments were observed by a fluorescent microscope (KEYENCE Biozero BZ-8000, Osaka, Japan).

Real-Time PCR Total RNA was isolated from ear biopsy samples, in the TPA-induced mouse ear edema model, using TRIzol $^{\circledR}$ Reagent according to the manufacturer's protocol. The RNA $(0.5 \mu \mathrm{g})$ was subsequently reverse-transcribed using ReverTra Ace ${ }^{\circledR}$ qPCR RT Master Mix (TOYOBO, Osaka, Japan). The expression levels of interleukin (IL)-6, tumor necrosis factor (TNF)- $\alpha$, cyclooxygenase (COX)-2 and IL- $1 \beta$ in the ear of the tested animals were determined using a real time PCR assay. Real-time PCR was performed on a CFX96 ${ }^{\mathrm{TM}}$ Real-Time PCR (Bio-Rad, Tokyo, Japan) using $1 \mu \mathrm{L}$ of $\mathrm{cDNA}$ for each sample. THUNDERBIRD ${ }^{\mathrm{TM}}$ SYBR $^{\circledR}$ qPCR Mix (TOYOBO) was used to detect products, and $10 \mu \mathrm{M}$ concentrations of the following primers were used: mouse glyceraldehyde-3-phosphate dehydrogenase (GAPDH) forward: 5'-GGT GAA GGTCGG TGT GAA CGG ATT-3', mouse GAPDH reverse: 5'-AAT GCCAAA GTTGTCATGGATGACC-3', mouse IL-6 forward: 5'-TGG AGT CAC AGA AGG AGT GGC TAA G-3', mouse IL-6 reverse: 5'-TCT GAC CAC AGT GAG GAA TGT CCA C-3', mouse COX-2 forward: 5'-AGC ACT GCA TCC TGC CAGCTC-3', mouse COX-2 reverse: 5'-AGA GGA CTG TGG CTC TGA CC-3', mouse TNF- $\alpha$ forward: $5^{\prime}$-AAC ATCCAACCT TCCCAAACG-3', mouse TNF- $\alpha$ reverse: 5'-CTC TTA ACC CCC GAA CTC CCA G-3', mouse IL-1 $\beta$ forward: 5'-AAA CCT CTT CGA GGC ACA AG-3', mouse IL- $1 \beta$ reverse: 5'-GTT TAGGGCCAT CAGCTT CA-3'. The relative amount of cDNA in each sample was normalized using GAPDH, and the melting curve was used to verify specificity. PCR was set at $95^{\circ} \mathrm{C}$ initially for $30 \mathrm{~s}$, followed by 40 cycles of $95^{\circ} \mathrm{C} \times 15 \mathrm{~s}, 55^{\circ} \mathrm{C} \times 15 \mathrm{~s}, 72^{\circ} \mathrm{C} \times 45 \mathrm{~s}$. The relative amount of cDNA in each sample was normalized using GAPDH, and the melting curve was used to verify specificity.

Cytotoxicity of Sacran Solutions in HaCaT Human Keratinocyte Cell Line $\mathrm{HaCaT}$ cells were seeded at $5 \times 10^{4}$ cells onto 96-well microplate (Iwaki, Tokyo, Japan), and incubated for $24 \mathrm{~h}$. Cells were washed once with phosphatebuffered saline (PBS, $\mathrm{pH}$ 7.4), and then incubated for $48 \mathrm{~h}$ with $200 \mu \mathrm{L}$ of Dulbecco's modified Eagle's medium (DMEM) containing sacran $(0.001,0.01,0.1$ or $0.25 \%(\mathrm{w} / \mathrm{v}))$ or Tween 20 at $37^{\circ} \mathrm{C}$. After washing with PBS, $100 \mu \mathrm{L}$ of fresh Hanks' balanced salt solution (HBSS, $\mathrm{pH} 7.4$ ) and $10 \mu \mathrm{L}$ of WST-1 reagent were added. After incubation for $30 \mathrm{~min}$, the absorbance at $450 \mathrm{~nm}$ against a reference wavelength of $655 \mathrm{~nm}$ was measured with a microplate reader (Bio-Rad Model 550).

Statistical Analysis Data are presented as the mean \pm standard error (S.E.). Significant differences between experimental values were determined using Scheffe's test, with $p<0.05$ considered significance.

\section{RESULTS}

Effects of Sacran on Carrageenan-Induced Acute Edema in Rat Paws In order to examine the anti-inflammatory activity of sacran in acute-phase inflammation in vivo, a carrageenan-induced paw edema experiment was conducted. The results revealed that sacran solutions showed a remarkable inhibitory effect in carrageenan-induced paw edema after carra- 
geenan injection (Fig. 1). The time dependent curve illustrated a continuous rise in paw swelling (\%) till $6 \mathrm{~h}$ after carrageenan injection. On the other hand, sacran solutions exhibited the bell shaped effect of down-regulating carrageenan-induced paw swelling, compared with the negative control group which was treated with normal saline only. Additionally, $0.05 \%(\mathrm{w} / \mathrm{v})$ sacran solution was the most effective concentration which was superior to BPAA, as a reference NSAID in edema inhibition.

Effects of Sacran on Histological Changes Associated with Carrageenan-Induced Rat Paw Edema Figure 2 shows the representative photos from the histological changes in the paw tissues following the subplantar injection of carrageenan and the anti-inflammatory effects of sacran solution $(0.05 \%(\mathrm{w} / \mathrm{v}))$. The carrageenan inflammatory response was found to be associated with a notable decrease in the epidermal layer thickness along with invasive infiltration of neutrophils in the dermis. In contrast, the treatment of animals with $0.05 \%(\mathrm{w} / \mathrm{v})$ sacran solution exhibited a conspicuous preservation of epidermis thickness as well as a decrease in neutrophils infiltration.

Skin Permeability of DMEQ-Labeled Sacran in Car-

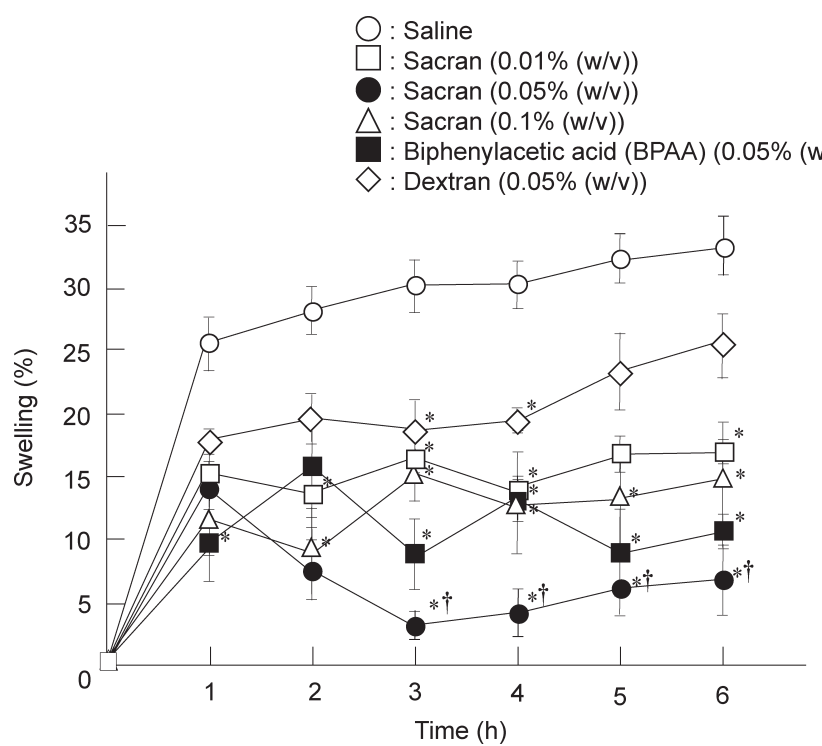

Fig. 1. Effects of Sacran on Carrageenan-Induced Acute Edema in Rat Paws

Various sacran solutions were administered every $1 \mathrm{~h}$ after subcutaneous injection of $\lambda$-carrageenan solution to rat paws. Each point represents the mean \pm S.E. of 5-20 experiments. ${ }^{*} p<0.05$, compared with saline. ${ }^{\dagger} p<0.05$, compared with dextran.
rageenan-Induced Rat Paw Edema Model To reveal the permeation of sacran into the skin, carrageenan-induced rat paw skin was cross sectioned after $3 \mathrm{~h}$ treatment with DMEQlabeled sacran. As illustrated in Fig. 3, the fluorescence of labeled sacran was observed in the epidermis and dermis layers, indicating the penetration ability of sacran in carrageenaninduced rat paw edema. Meanwhile, in the case of normal rat skin, the fluorescence was noticed only in the stratum corneum layer, suggesting negligible penetration of sacran.

Effects of Sacran on Kaolin-Induced Rat Paw Edema Kaolin is one of the few phlogistic agents which mediate kinins and it does so mainly by prostaglandins. ${ }^{15)}$ The results of the kaolin-induced rat paw edema are shown in Fig. 4. In this experimental model, the inflammatory edema start to induce after $1 \mathrm{~h}$ and peaked at $5 \mathrm{~h}$ after kaolin injection. Topical application of sacran solutions at concentrations of 0.01 and $0.05 \%$ (w/v) significantly suppressed the edema formation, compared with that of $0.1 \%(\mathrm{w} / \mathrm{v})$.

Effects of Sacran on Dextran-Induced Rat Paw Edema Dextran is a well-known phlogistic agent which induces paw edema as a result of liberation of histamine and serotonin from mast cells. ${ }^{16)}$ The anti-inflammatory effect of sacran against dextran-induced rat paw edema is illustrated in Fig. 5. The results demonstrated that the administration of sacran solutions significantly inhibited dextran-induced paw edema throughout the duration of the study in a concentrationdependent manner with the optimum effective concentration at $0.05 \%(\mathrm{w} / \mathrm{v})$.

Effects of Sacran on TPA-Induced Mouse Ear Edema Figure 6 demonstrates the effects of the topical administration of sacran solutions on TPA-induced mouse ear edema. The data indicated that application of TPA promoted an increase in ear thickness. Contrarily, a sacran solution $(0.05 \%(\mathrm{w} / \mathrm{v}))$ showed the maximum inhibitory effect on ear swelling in the TPA-induced ear edema model.

Effects of Sacran on mRNA Expression Levels of COX-2 and Inflammatory Cytokines in TPA-Induced Mouse Ear Edema Topical application of TPA, a specific activator of protein kinase $\mathrm{C}(\mathrm{PKC})$, represents a suitable skin inflammation model for assessing the topical anti-inflammatory agents. Next, we investigated the effect of $0.05 \%$ (w/v) sacran solution on the mRNA expression levels of COX-2 and various cytokines such as TNF- $\alpha$, IL- 6 , and IL- $1 \beta$ after $6 \mathrm{~h}$ in TPAinduced mice model. These results indicated that $0.05 \%(\mathrm{w} / \mathrm{v})$ sacran solution significantly suppressed those mRNA expressions, compared with TPA alone (Fig. 7).

\section{Control}

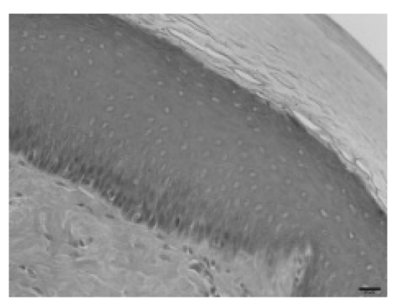

Carrageenan alone

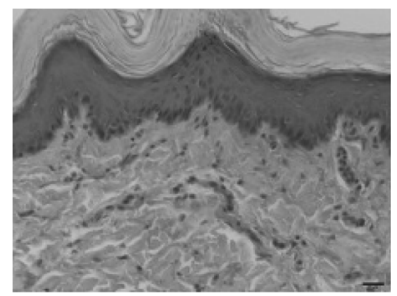

$\lambda$-Carrageenan

+ Sacran $(0.05 \%(w / v))$

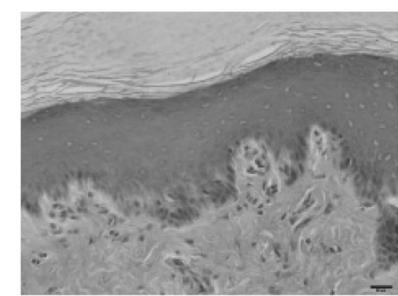

Fig. 2. Hematoxylin \& Eosin Staining of Carrageenan-Induced Acute Edema in Rat Paws

Sacran solution $(0.05 \%(\mathrm{w} / \mathrm{v}))$ was administered every $1 \mathrm{~h}$ after subcutaneous injection of $\lambda$-carrageenan solution to a rat paw. A paw tissue was resected $3 \mathrm{~h}$ after subcutaneous injection of $\lambda$-carrageenan solution. The experiments were performed independently three times, and the representative images are shown. 


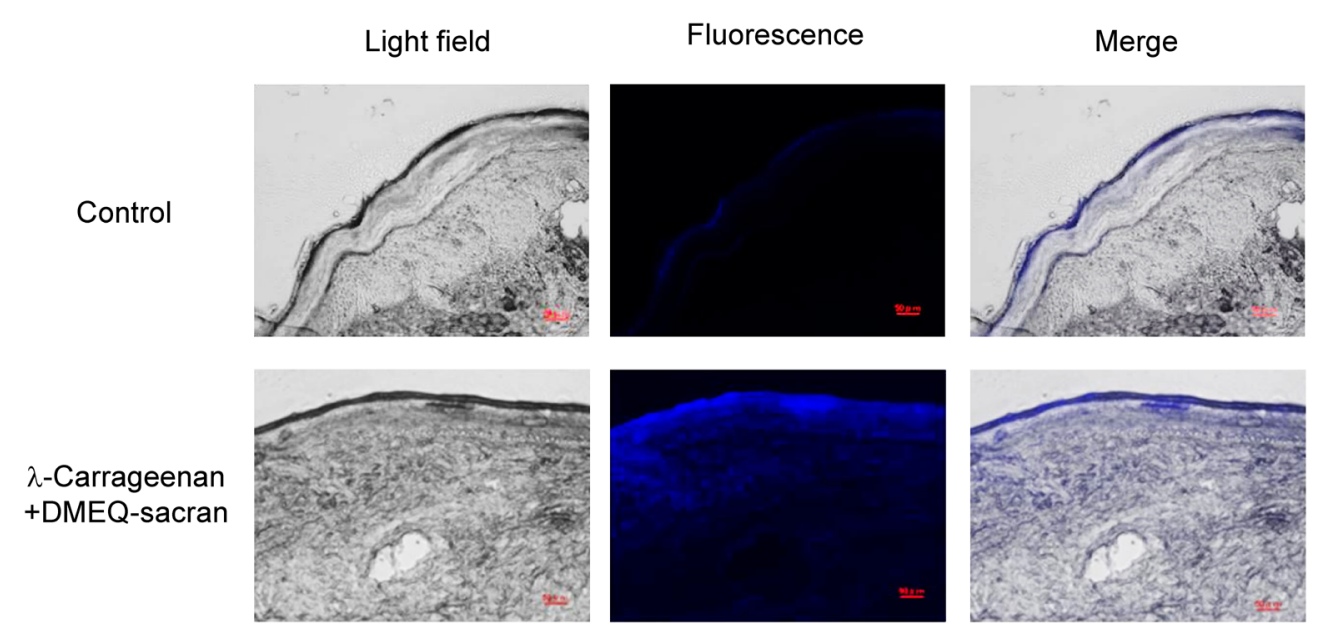

Fig. 3. Photographs of Tissue Slices of Rat Paw after Treatment of DMEQ-Labeled Sacran

Labeled sacran solution $(0.05 \%(\mathrm{w} / \mathrm{v}))$ was administered every $1 \mathrm{~h}$ after subcutaneous injection of $\lambda$-carrageenan solution to a rat paw. After $3 \mathrm{~h}$, resected segments were observed with a fluorescent microscope.

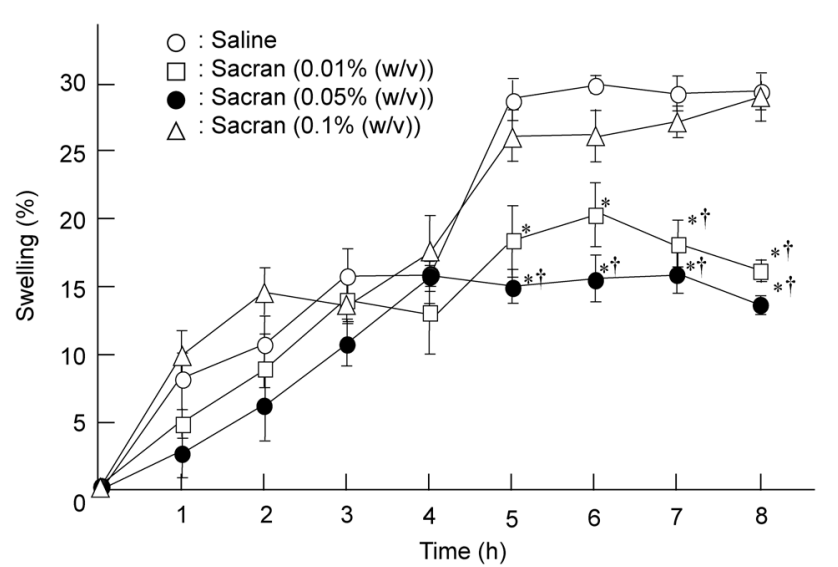

Fig. 4. Effects of Sacran on Kaolin-Induced Acute Edema in Rat Paws

The sacran solution $(0.01,0.05,0.1 \%(\mathrm{w} / \mathrm{v}))$ was administered every $1 \mathrm{~h}$ after subcutaneous injection of kaolin solution to rat paws. Each point represents the mean \pm S.E. of $5-20$ experiments. ${ }^{*} p<0.05$, compared with saline. ${ }^{\dagger} p<0.05$, compared with sacran $(0.1 \%(\mathrm{w} / \mathrm{v}))$.

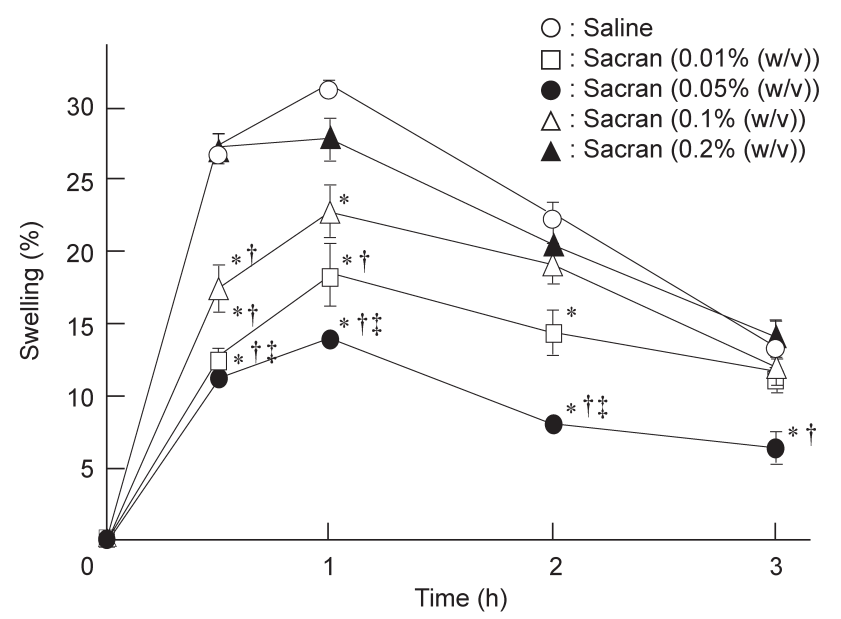

Fig. 5. Effects of Sacran on Dextran-Induced Acute Edema in Rat Paws

The sacran solution $(0.01,0.05,0.1,0.2 \%(\mathrm{w} / \mathrm{v}))$ was administered every $1 \mathrm{~h}$ after subcutaneous injection of dextran solution to rat paws. Each point represents the mean \pm S.E. of 5-6 experiments. $* p<0.05$, compared with saline. $t p<0.05$, compared with sacran $(0.1 \%(\mathrm{w} / \mathrm{v})) .{ }^{\dagger} p<0.05$, compared with sacran $(0.2 \%(\mathrm{w} / \mathrm{v}))$.

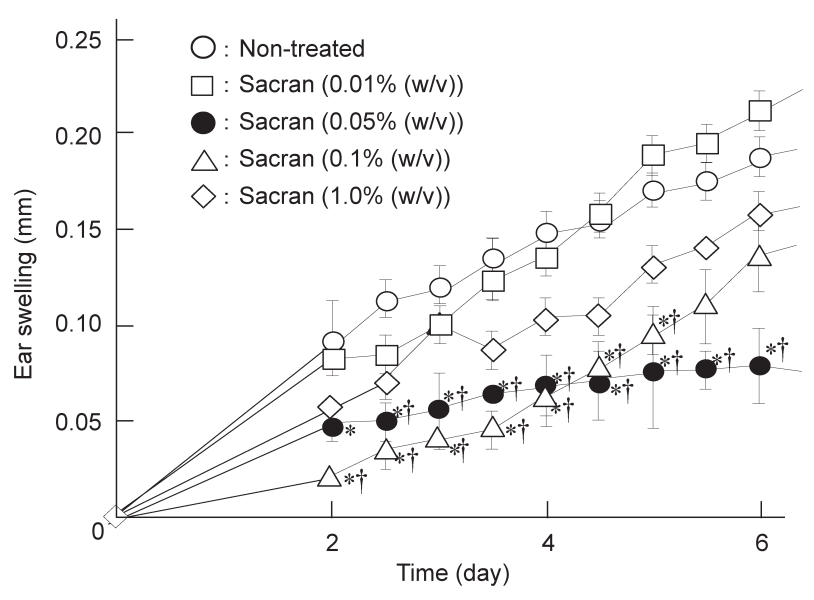

Fig. 6. Effects of Sacran on TPA-Induced Skin Inflammation in Mice

Three microliters of TPA $(1 \mathrm{mg} / \mathrm{mL})$ was treated on mice ear, and then $40 \mu \mathrm{L}$ of sacran solutions $(0.01,0.05,0.1,1.0 \%(\mathrm{w} / \mathrm{v}))$ were applied to the ear. Ear swelling was measured by using a thickness gauge. Each point represents the mean \pm S.E. of 5-17 experiments. ${ }^{*} p<0.05$, compared with Non-treated. ${ }^{\dagger} p<0.05$, compared with $0.01 \%(\mathrm{w} / \mathrm{v})$ sacran

Cytotoxicity of Sacran Solutions in HaCaT Human Keratinocyte Cell Line Keratinocyte is considered to be one of the major cell types involved in skin inflammatory responses. ${ }^{17)}$ To reveal the safety of sacran solutions, we examined the cytotoxicity of sacran solutions in HaCaT cells after incubation for $48 \mathrm{~h}$ by the WST-1 method (Fig. 8). As a result, sacran solutions showed negligible cytotoxicity up to a concentration of $0.25 \%(\mathrm{w} / \mathrm{v})$.

\section{DISCUSSION}

This study highlights the topical anti-inflammatory effects of sacran polysaccharide on various experimental inflammatory models induced by different phlogistic agents as useful pharmacological tools for the investigation of new anti-inflammatory substances, particularly of plant origin, to treat inflammatory skin disorders.

Firstly, carrageenan-induced rat paw edema was used as a model of acute inflammation for assessing the anti-inflammatory effects of sacran. Subplantar injection of carrageenan 

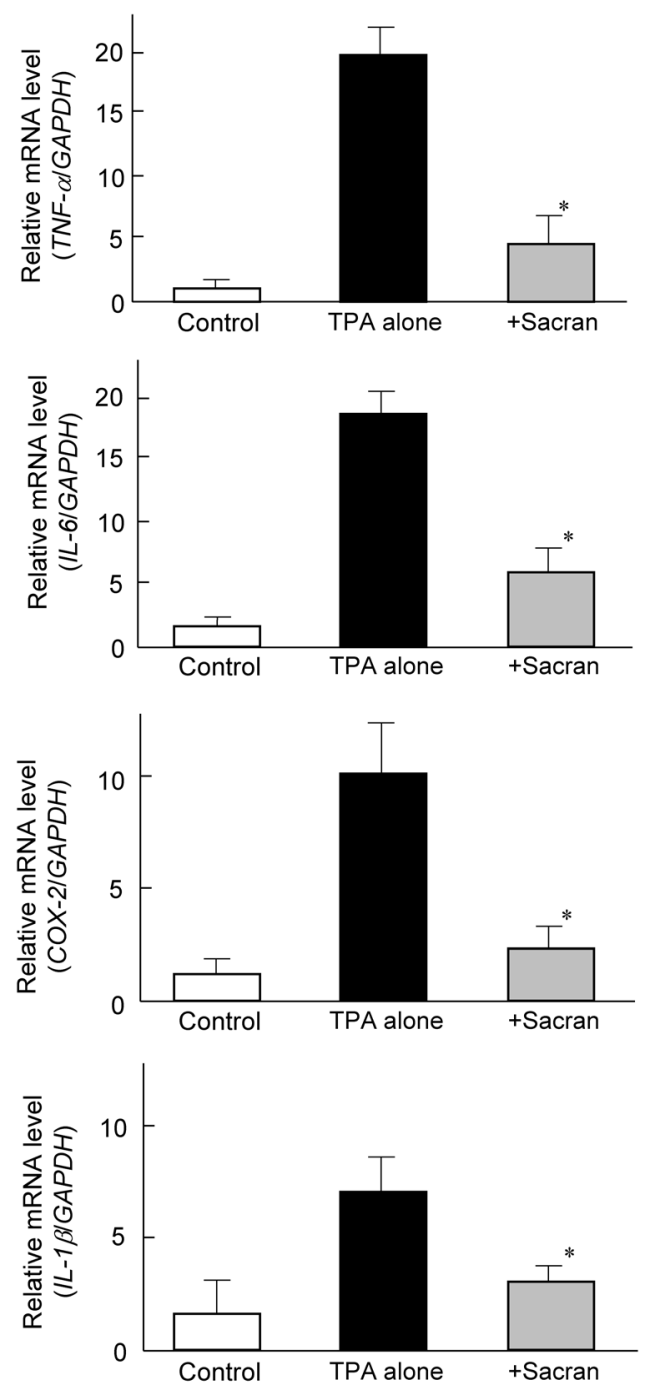

Fig. 7. Effects of Sacran on mRNA Levels on TPA-Induced Skin Inflammation in Mice

Three microliters of TPA $(1 \mathrm{mg} / \mathrm{mL})$ was treated on mice ear, and then $40 \mu \mathrm{L}$ of $0.05 \%(\mathrm{w} / \mathrm{v})$ sacran solution was applied to the ear. After $6 \mathrm{~h}$, the ears were collected. Total RNA was extracted by TRIzol ${ }^{\circledR}$ reagent, and then the mRNA levels were determined by real-time PCR. Each value represents the mean \pm S.E. of 3-4 experiments. ${ }^{*} p<0.05$, compared with TPA alone.

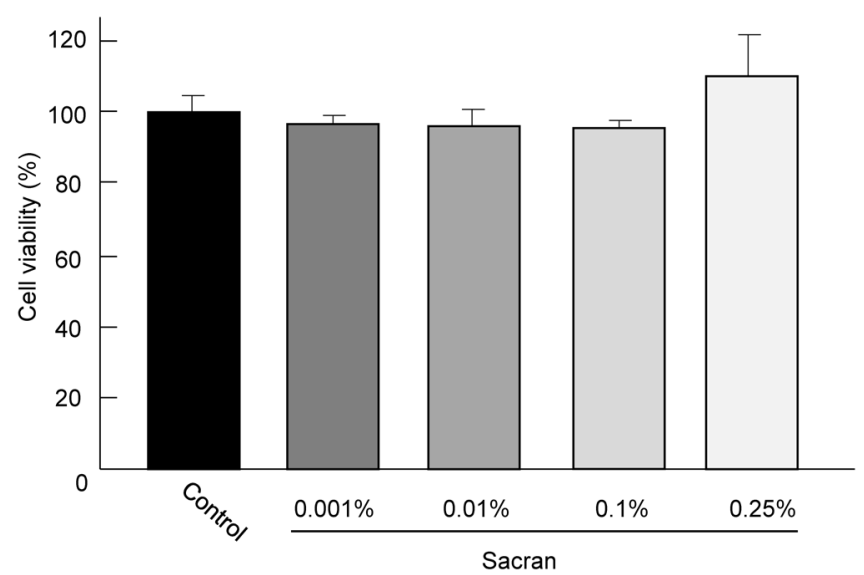

Fig. 8. Cytotoxicity of Sacran Solutions in HaCaT Cells

Cells were incubated with sacran solutions for $48 \mathrm{~h}$. Cell viability was assayed by the WST-1 method. The concentrations of sacran were $0.001,0.01,0.1$ and $0.25 \%$ $(\mathrm{w} / \mathrm{v})$. Each value represents the mean \pm S.E. of 5-6 experiments. produces a biphasic response. The initial phase results from release of histamine, serotonin, and kinins for the first $2 \mathrm{~h}$; meanwhile, the subsequent phase is attributed to the release of prostaglandins as well as neutrophil infiltration. ${ }^{18)}$ In the present study, topical application of $0.05 \%(\mathrm{w} / \mathrm{v})$ sacran solution significantly reduced two critical events related to skin inflammatory response induced by carrageenan; both paw edema and neutrophils infiltration in rat paw tissues, as compared with the controls (Figs. 1, 2). This effect was similar to that of BPAA as a reference drug at the same concentration. Nonsteroidal anti-inflammatory drugs such as BPAA, the active metabolite of fenbufen, act as potent inhibitors of prostaglandins synthesis which are important mediators of acute inflammation. ${ }^{19-22)}$ Based on these findings, the ability of sacran to reduce edema formation in carrageenan-induced rats might be attributed to its inhibitory effect on prostaglandin synthesis. A similar proposal was reported for the anti-inflammatory activity of fucan, a sulphated polysaccharide, against carrageenaninduced paw edema in rats. ${ }^{23}$ Therefore, the prostaglandins levels in the skin should be determined hereafter.

It is worth noting that the fluorescence of labeled sacran was observed in the epidermis and dermis layers in carrageenan-induced rat paw edema. Meanwhile, the fluorescence was noticed only in the stratum corneum layer in normal rat skin (Fig. 3). These results suggest that sacran can penetrate into the epidermis of skin in edema.

A second model of acute inflammation used in our study was induced by kaolin. Sacran solutions at concentrations of 0.01 and $0.05 \%(\mathrm{w} / \mathrm{v})$ significantly elucidated a prominent inhibition of the kaolin-induced rat paw edema throughout the duration of the study (Fig. 4). It has been reported that the inflammatory response to kaolin induction was mediated by kinins and mainly by prostaglandins. ${ }^{15)}$ Therefore, our data further suggest the anti-inflammatory activity of sacran which could be at least partially through inhibition of prostaglandins biosynthesis.

Dextran is a well-known phlogistic agent which induces paw edema as a result of liberation of histamine and serotonin from mast cells. ${ }^{16)}$ Sacran solution $(0.05 \%$ (w/v)) efficiently suppressed the dextran-induced paw edema (Fig. 5). Previously, we demonstrated that sacran solution suppressed the $\beta$-hexosaminidase release in RBL-2H3 cells, which are now known to be an analog of rat mucosal mast cells, indicating the inhibition of mast cell degranulation. ${ }^{24)}$ These results suggest that the anti-inflammatory effects of sacran may contribute to inhibition of not only prostaglandins but also other inflammatory mediators.

The inflammatory features of TPA-induced mouse ear edema are characterized by edema, epidermal hyperplasia and infiltration of inflammatory cells as well as liberation of inflammatory mediators as COX-2, IL- 6 , TNF- $\alpha$, and IL-1 $\beta$ in mouse skins. ${ }^{25-29)}$ Our data demonstrated that topical application of $0.05 \%(\mathrm{w} / \mathrm{v})$ sacran solution significantly suppressed TPA-induced ear edema and mRNA expression levels of COX-2 as well as pro-inflammatory cytokines such as TNF- $\alpha$, IL- $1 \beta$, and IL-6 (Figs. 6, 7). These results suggest that the anti-inflammatory effects of sacran solution may be a consequence of down-regulation of TPA-induced expression of COX-2, IL- $1 \beta$, IL- 6 , and TNF- $\alpha$. The detailed mechanism remains to be further elucidated.

Sacran is a unique cyanobacteria-derived glycosamino- 
glycanoid. The anti-inflammatory effect displayed by sacran could be related to the similarity of its chemical structure to that of glycosaminoglycans, sulfated polysaccharides, which possess numerous bioactivities including an anti-inflammatory effect. $^{30-34)}$ The high sulfate and carboxyl group content of glycosaminoglycans permits them to interact with a wide range of proteins, enzymes, cytokines, chemokines, lipoproteins, and adhesion molecules. ${ }^{35-38)}$ Also, Ngatu et al. reported that epicutaneous application of sacran to 2,4,6-trinitrochlorobenzene-induced $\mathrm{NC} / \mathrm{Nga}$ mice significantly inhibited the development of allergic dermatitis skin lesions, and decreased the number of scratching behavior episodes by improving the stability, elasticity, and hydration of skin barrier as well as inhibiting the production of T-helper 2 (Th2) cytokines (IL-4, IL-5), Th1 cytokines (TNF- $\alpha$, interferon- $\gamma$ (IFN- $\gamma$ )) and inflammatory chemokines such as monocyte chemotactic protein-1 (MCP-1) and eotaxin; thus, inhibiting immunoglobulin $\mathrm{E}$ (IgE) and eosinophilic infiltration in mice. ${ }^{13)}$ These unique properties exhibited by sacran are not common in most sulfated polysaccharides.

In the view of the data mentioned above, the anti-inflammatory activity of sacran solutions in this study was in a concentration-dependent manner, with the optimum effective concentrations at 0.01 and $0.05 \%(\mathrm{w} / \mathrm{v})$. This effect is related to chain properties such as electric charges or the chain conformation. Recently, Mitsumata et al. demonstrated that, in diluted regime less than $0.09 \%(\mathrm{w} / \mathrm{w})$, the single chain of sacran occupies a spherical free volume with a diameter of the fluctuation length. Meanwhile, at the concentrated regime the sacran chain loses its electric charges and forms double helixes at $0.1 \%(\mathrm{w} / \mathrm{w})$ due to reduction in the electric repulsive force on the saccharide chain. In addition, it forms a weak gel at $0.2 \%$ (w/w). A macroscopic domain of liquid crystals observed at concentrations over $0.2 \%(\mathrm{w} / \mathrm{w})$ is probably due to the hydrophobic cross-linking points between double helixes, indicating their extremely persistent length. ${ }^{39)}$ As a consequence, from a biological point of view, the gelation mechanism of sacran chain is so critical that it may affect its molecular structure, size and length and subsequently its cutaneous penetration through the inflamed tissue.

\section{CONCLUSION}

Currently, scientific research is focused on the discovery of new natural products with anti-inflammatory activity. In the present study, sacran solution provoked potent antiinflammatory effects when applied topically to different acute and chronic inflammatory animal models. The probable antiinflammatory mechanism of sacran involves numerous targets, resulting in suppression of essential inflammatory mediators in the cutaneous tissue. Nevertheless, further elaborate studies are required to determine the precise mechanism of inflammatory action of sacran and ascertain its clinical use.

Conflict of Interest The authors have no conflict of interest directly relevant to the content of this article. S. Kaneko is CEO of Green Science Material Inc.

\section{REFERENCES}

1) Bos JD. The skin as an organ of immunity. Clin. Exp. Immunol.,
107 (Suppl. 1), 3-5 (1997).

2) Hengge UR, Ruzicka T, Schwartz RA, Cork MJ. Adverse effects of topical glucocorticosteroids. J. Am. Acad. Dermatol., 54, 1-15, quiz, 16-18 (2006).

3) Schoepe S, Schacke H, May E, Asadullah K. Glucocorticoid therapy-induced skin atrophy. Exp. Dermatol., 15, 406-420 (2006).

4) Bellik Y, Boukraâ L, Alzahrani HA, Bakhotmah BA, Abdellah F, Hammoudi SM, Iguer-Ouada M. Molecular mechanism underlying anti-inflammatory and anti-allergic activities of phytochemicals: an update. Molecules, 18, 322-353 (2013).

5) Yuan G, Wahlqvist ML, He G, Yang M, Li D. Natural products and anti-inflammatory activity. Asia Pac. J. Clin. Nutr., 15, 143-152 (2006).

6) Averbeck M, Gebhardt CA, Voigt S, Beilharz S, Anderegg U, Termeer CC, Sleeman JP, Simon JC. Differential regulation of hyaluronan metabolism in the epidermal and dermal compartments of human skin by UVB irradiation. J. Invest. Dermatol., 127, 687-697 (2007).

7) Yanase Y, Hiragun T, Uchida K, Ishii K, Oomizu S, Suzuki H, Mihara S, Iwamoto K, Matsuo H, Onishi N, Kameyoshi Y, Hide M. Peritoneal injection of fucoidan suppresses the increase of plasma IgE induced by OVA-sensitization. Biochem. Biophys. Res. Commun., 387, 435-439 (2009).

8) Okajima MK, Bamba T, Kaneso Y, Hirata K, Fukusaki E, Kajiyama S, Kaneko T. Supergiant ampholytic sugar chains with imbalanced charge ratio form saline ultra-absorbent hydrogels. Macromolecules, 41, 4061-4064 (2008).

9) Okajima MK, Higashi T, Asakawa R, Mitsumata T, Kaneko D, Kaneko T, Ogawa T, Kurata H, Isoda S. Gelation behavior by the lanthanoid adsorption of the cyanobacterial extracellular polysaccharide. Biomacromolecules, 11, 3172-3177 (2010).

10) Okajima MK, Kaneko D, Mitsumata T, Kaneko T, Watanabe J. Cyanobacteria that produce megamolecules with efficient selforientations. Macromolecules, 42, 3057-3062 (2009).

11) Okajima MK, Miyazato S, Kaneko T. Cyanobacterial megamolecule sacran efficiently forms LC gels with very heavy metal ions. Langmuir, 25, 8526-8531 (2009).

12) Okajima MK, Nakamura M , Mitsumata T, Kaneko T. Cyanobacterial polysaccharide gels with efficient rare-earth-metal sorption. Biomacromolecules, 11, 1773-1778 (2010).

13) Ngatu NR, Okajima MK, Yokogawa M, Hirota R, Eitoku M, Muzembo BA, Dumavibhat N, Takaishi M, Sano S, Kaneko T, Tanaka T, Nakamura H, Suganuma N. Anti-inflammatory effects of sacran, a novel polysaccharide from Aphanothece sacrum, on 2,4,6-trinitrochlorobenzene-induced allergic dermatitis in vivo. Ann. Allergy Asthma Immunol., 108, 117-122.e2 (2012).

14) Matsumoto K, Nishi K, Kikuchi M, Kadowaki D, Tokutomi Y, Tokutomi N, Nishi K, Suenaga A, Otagiri M. $\alpha_{1}$-Acid glycoprotein suppresses rat acute inflammatory paw edema through the inhibition of neutrophils activation and prostaglandin E2 generation. Biol. Pharm. Bull., 30, 1226-1230 (2007).

15) Lewis AJ, Cottney J, Nelson DJ. Mechanisms of phytohaemagglutinin-P-, concanavalin-A- and kaolin-induced oedemas in the rat. Eur. J. Pharmacol., 40, 1-8 (1976).

16) Rowley DA, Benditt EP. 5-Hydroxytryptamine and histamine as mediators of the vascular injury produced by agents which damage mast cells in rats. J. Exp. Med., 103, 399-412 (1956).

17) Murphy JE, Robert C, Kupper TS. Interleukin-1 and cutaneous inflammation: a crucial link between innate and acquired immunity. J. Invest. Dermatol., 114, 602-608 (2000).

18) Vinegar R, Schreiber W, Hugo R. Biphasic development of carrageenin edema in rats. J. Pharmacol. Exp. Ther., 166, 96-103 (1969).

19) Brooks PM, Day RO. Nonsteroidal antiinflammatory drugs-differences and similarities. N. Engl. J. Med., 324, 1716-1725 (1991).

20) Chiccarelli FS, Eisner HJ, Van Lear GE. Disposition and metabolism of fenbufen in several laboratory animals. Arzneimittelfors- 
chung, 30 (4A), 707-715 (1980).

21) Giuliano F, Warner TD. Origins of prostaglandin E2: involvements of cyclooxygenase (COX)-1 and COX-2 in human and rat systems. J. Pharmacol. Exp. Ther., 303, 1001-1006 (2002).

22) Kerwar SS. Pharmacologic properties of fenbufen. Am. J. Med., 75 (4B), 62-69 (1983).

23) Mohsin S, Kurup GM. Mechanism underlying the anti-inflammatory effect of sulphated polysaccharide from Padina tetrastromatica against carrageenan induced paw edema in rats. Biomedicine \& Preventive Nutrition, 1, 294-301 (2011).

24) Fukushima S, Motoyama K, Tanida Y, Higashi T, Ishitsuka Y, Kondo Y, Irie T, Tanaka T, Ihn H, Arima H. Clinical evaluation of novel natural polysaccharides sacran as a skincare material for atopic dermatitis patients. JCDSA, 6, 9-18 (2016).

25) De Vry CG, Valdez M, Lazarov M, Muhr E, Buelow R, Fong T, Iyer S. Topical application of a novel immunomodulatory peptide, RDP58, reduces skin inflammation in the phorbol ester-induced dermatitis model. J. Invest. Dermatol., 125, 473-481 (2005).

26) Ha HY, Kim Y, Ryoo ZY, Kim TY. Inhibition of the TPA-induced cutaneous inflammation and hyperplasia by EC-SOD. Biochem. Biophys. Res. Commun., 348, 450-458 (2006).

27) Murakawa M, Yamaoka K, Tanaka Y, Fukuda Y. Involvement of tumor necrosis factor (TNF)- $\alpha$ in phorbol ester 12-O-tetradecanoylphorbol-13-acetate (TPA)-induced skin edema in mice. Biochem. Pharmacol., 71, 1331-1336 (2006).

28) Stanley PL, Steiner S, Havens M, Tramposch KM. Mouse skin inflammation induced by multiple topical applications of $12-O$-tetradecanoylphorbol-13-acetate. Skin Pharmacol., 4, 262-271 (1991).

29) Xian YF, Lin ZX, Xu XY, Su ZR, Chen JN, Lai XP, Ip SP. Effect of Rhizoma Polygonati on 12-O-tetradecanoylphorbol-acetate-induced ear edema in mice. J. Ethnopharmacol., 142, 851-856 (2012).

30) Kuschert GS, Coulin F, Power CA, Proudfoot AE, Hubbard RE,
Hoogewerf AJ, Wells TN. Glycosaminoglycans interact selectively with chemokines and modulate receptor binding and cellular responses. Biochemistry, 38, 12959-12968 (1999).

31) Parish CR. The role of heparan sulphate in inflammation. Nat. Rev. Immunol., 6, 633-643 (2006).

32) Park HY, Han MH, Park C, Jin CY, Kim GY, Choi IW, Kim ND, Nam TJ, Kwon TK, Choi YH. Anti-inflammatory effects of fucoidan through inhibition of NF- $\kappa \mathrm{B}$, MAPK and Akt activation in lipopolysaccharide-induced BV2 microglia cells. Food Chem. Toxicol., 49, 1745-1752 (2011).

33) Volpi N. Therapeutic applications of glycosaminoglycans. Curr. Med. Chem., 13, 1799-1810 (2006).

34) Yamada S, Sugahara K. Potential therapeutic application of chondroitin sulfate/dermatan sulfate. Curr. Drug Discov. Technol., 5, 289-301 (2008).

35) Bernfield M, Gotte M, Park PW, Reizes O, Fitzgerald ML, Lincecum J, Zako M. Functions of cell surface heparan sulfate proteoglycans. Annu. Rev. Biochem., 68, 729-777 (1999).

36) Kawashima H, Atarashi K, Hirose M, Hirose J, Yamada S, Sugahara K, Miyasaka M. Oversulfated chondroitin/dermatan sulfates containing GlcA $\beta 1 / \mathrm{IdoA} \alpha 1 \rightarrow 3 \mathrm{GalNAc}(4,6-O$-disulfate) interact with L- and P-selectin and chemokines. J. Biol. Chem., 277, 12921-12930 (2002).

37) Salmivirta M, Lidholt K, Lindahl U. Heparan sulfate: a piece of information. FASEB J., 10, 1270-1279 (1996).

38) Taylor KR, Gallo RL. Glycosaminoglycans and their proteoglycans: host-associated molecular patterns for initiation and modulation of inflammation. FASEB J., 20, 9-22 (2006).

39) Mitsumata T, Miura T, Takahashi N, Kawai M, Okajima MK, Kaneko T. Ionic state and chain conformation for aqueous solutions of supergiant cyanobacterial polysaccharide. Phys. Rev. E Stat. Nonlin. Soft Matter. Phys., 87, 042607 (2013). 